\title{
Extracting and Analyzing Deep Learning Features for Discriminating Historical Art
}

\author{
Deep Learning Features and Art
}

\author{
Paul Rodriguez \\ San Diego Supercomputer Center University of California \\ San Diego, La Jolla, CA USA \\ p4rodriguez@ucsd.edu \\ Alison Langmead \\ University of Pittsburgh Pittsburgh, PA, USA \\ adlangmead@pitt.edu
}

\author{
Alan Craig \\ University of Illinois, Urbana-Champaign, Urbana \\ Champaign, Illinois, USA \\ a-craig@illinois.edu \\ Christopher J. Nygren \\ University of Pittsburgh Pittsburgh, PA, USA \\ cnygren@pitt.edu
}

\begin{abstract}
Art historians are interested in possible methods and visual criteria for determining the style and authorship of artworks. One approach, developed by Giovanni Morelli in the late nineteenth century, focused on abstracting, extracting and comparing details of recognizable human forms, although he never prescribed what exactly to look for. In this work, we asked what could a contemporary method like convolution networks contribute or reveal about such a humanistic method that is not fully determined, but that is also so clearly aligned with computation? Convolution networks have become very successful in object recognition because they learn general features to distinguish and classify large sets of objects. Thus, we wanted to explore what features are present in these networks that have some discriminatory power for distinguishing paintings. We input the digitized art into a large-scale convolutional network that was pre-trained for object recognition from naturalistic images. Because we do not have labels, we extracted activations from the network and ran K-means clustering. We contrasted and evaluated discriminatory power between shallow and deeper layers. We also compared predetermined features from standard computer vision techniques of edge detection. It turns out that the deep network individual feature maps are highly generic and do not easily map onto obvious authorship interpretations, but in the aggregate can have strong discriminating power that are intuitive. Although this does not directly test issues of attribution, the application can inform humanistic perspectives regarding what counts as features that make up visual elements of paintings.
\end{abstract}

\section{CCS CONCEPTS}

- Applied deep learning; • Digital Humanities; • Clustering;

Permission to make digital or hard copies of all or part of this work for personal or classroom use is granted without fee provided that copies are not made or distributed for profit or commercial advantage and that copies bear this notice and the full citation on the first page. Copyrights for components of this work owned by others than the author(s) must be honored. Abstracting with credit is permitted. To copy otherwise, or republish, to post on servers or to redistribute to lists, requires prior specific permission and/or a fee. Request permissions from permissions@acm.org.

PEARC '20, fuly 26-30, 2020, Portland, OR, USA

(C) 2020 Copyright held by the owner/author(s). Publication rights licensed to ACM. ACM ISBN 978-1-4503-6689-2/20/07 .. \$15.00

https://doi.org/10.1145/3311790.3399611

\section{KEYWORDS}

Digital humanities, deep convolution network, clustering

\section{ACM Reference Format:}

Paul Rodriguez, Alan Craig, Alison Langmead, and Christopher J. Nygren. 2020. Extracting and Analyzing Deep Learning Features for Discriminating Historical Art: Deep Learning Features and Art. In Practice and Experience in Advanced Research Computing (PEARC '20), fuly 26-30, 2020, Portland, OR, USA. ACM, New York, NY, USA, 6 pages. https://doi.org/10.1145/3311790. 3399611

\section{INTRODUCTION}

Art historians sometimes disagree on the authorship of even wellstudied paintings [1]. The issue is often not a question of forgery, but rather that the painting could have been painted by the master, or the student, or even both. Giovanni Morelli in the late nineteenth century, presented a semi-formal approach to extract and compare visual elements of parts of paintings that included human forms, such as eyes, mouths, or hands. He gave procedures and examples but he never prescribed what features to use, which still left the task quite subjective. If art historians cannot satisfactorily agree on what features are most relevant, perhaps there are other, more empirical ways, that computers can be enlisted to bring clarity to this problem [2]. It is plausible to hypothesize that computers might do a better job of directly, and accurately, attributing artists to artworks, if only we could tell the computer what to look for or what to learn. This work is an investigation into how deep learning networks could inform the humanist about what may or may not be represented in state-of-the-art computational methods.

Computer vision techniques for object discrimination rely on either predetermined features or feature discovery. Predetermined features include techniques such as, edge detection, histogramof-gradients of pixel intensity, and entropy. On the other hand, convolutional neural networks $(\mathrm{CNN})$ learn filters that act as feature detectors for the training task, which is sometimes called feature discovery. In CNNs trained for object recognition lower layers seem to reflect texture, color, and intensity variations, and higher layers reflect object-like elements, like shapes. Thus, in principle, networks that can recognize many objects consist of general-purpose features that can discriminate object-like combinations of elements such as color, edges, textures, or lines. 


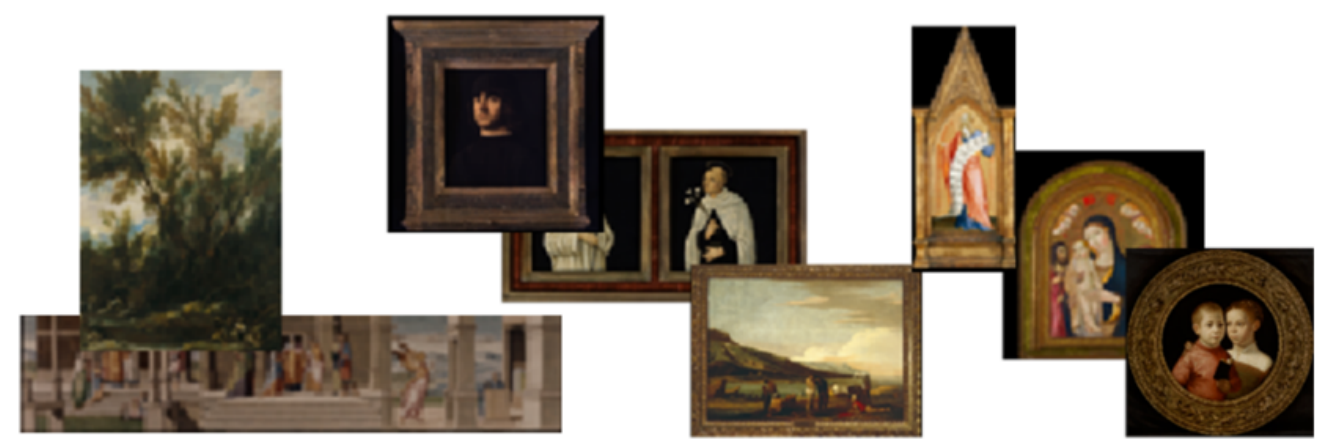

Figure 1: Digital photos of the art work shows a variety of frames, shapes, and occasional extra background behind the frame.

Some of the images in our corpus have specific author attributions, while many others are more generally associated with regions, periods, and artistic schools. To make particular labels describing styles or authors who are in the same or different regions, periods, or schools would therefore require us to define what features are important. That would be completely counter to our goal of discovering if the computer could reveal to us useful features for the attribution of artworks. Although we do not have labels for supervised training, we can still extract activation maps from the convolution layers, which reflect the learned features. Those activations feature maps are then used as input vectors for unsupervised clustering. The clusters, and the vector values that define those clusters, will then form the basis of how we think about what deep learning can offer the humanist.

In the methods Section 2 below, we first describe the image preparation, the convolution network, and feature map extraction steps. Then in the results Section 3, we apply K-mean clustering, analyze discriminatory power of features from the network, and compare that to predetermined features. Section 4 discusses implications, interpretations, and future work.

\section{DATA PREPARATION AND MODELING}

Convolution networks in object recognition are often trained with the kinds of digital images that one expects to find on the internet, which are typically photographs of everyday life, not digitized historical paintings. Here we describe the choices and steps for preparing images for input to a network. We also describe the network choices and trade- offs for extracting information from the feature maps that feed into clustering. The main goal is to extract useful information that captures the network representations without biasing the cluster procedure. All together, we have a pipeline of preprocessing images for the $\mathrm{CNN}$, extracting feature maps, preprocessing feature maps for clustering, and then clustering.

\subsection{Images}

The team acquired approximately 1866 high resolution images, most with $4 \mathrm{~K}$ to $5 \mathrm{~K}$ heights and widths, from the collection of the Samuel H. Kress Foundation, who were very generous in sharing their collection of digital images. Images had to be resized and cropped at 224x224 pixels for input to the VGG19 model. However, this turned out to be non-trivial because many images ( 300-400) had frames in the digital image (Figure 1).

After some initial testing it was determined that the frames were relatively prominent for the $\mathrm{CNN}$ (e.g. clusters seemed to be all about the frame), and this was especially the case for rounded and pointed frames. Therefore, we tried out various resizing and cropping procedures, but nothing could handle the variety and inconsistency of frames sizes, thickness, or shape. It would have been very time consuming to draw out croppings or size-up boxes by hand, and/or train a new network to segment such different kinds of frames. Instead we compromised and chose a semi-manual method. We applied 4 resizings (shortest-side pixel length: 224 to 450 ) combined with 3 croppings (center, $+/$ - off center), and then simply selected the best combination manually, as shown in Figure 2 below. For some images it was too difficult to get reasonable square croppings (especially the triangular frames, the frames with panels, or those with poor digitization), which left about 1820 images.

\subsection{Convolution Network}

Several large-scale networks that have been pre-trained to categorize objects are readily available in deep learning libraries such as CAFFE and KERAS. We chose the VGG 19 convolution network (from Oxford Visual Geometry Group [3]) because it has been successfully applied to other tasks, such as image segmentation, which suggests that it has both fine detail filters and general filters that help produce detailed mask images.

In Figure 3 we show the architecture of the VGG 19 network. There are 5 blocks of convolution layers in which the last layer in the block is a max-pooling layer. This layer is a down-sampling summary that takes a maximum over a pre-specified pixel window. For example, the image input size is $224 \times 224 \times 3$, the first block has $64224 \times 224$ convolution maps that are then down-sampled to 64 112x112 max-pooled maps.

\subsection{From Feature Map Activity to Cluster Input}

As mentioned above, we do not have labels, or well-defined classes for the images. In fact, for the experts, trying to come up with labels is somewhat fraught with difficulties in defining attributes and making assumptions. Therefore, we employ the convolution 


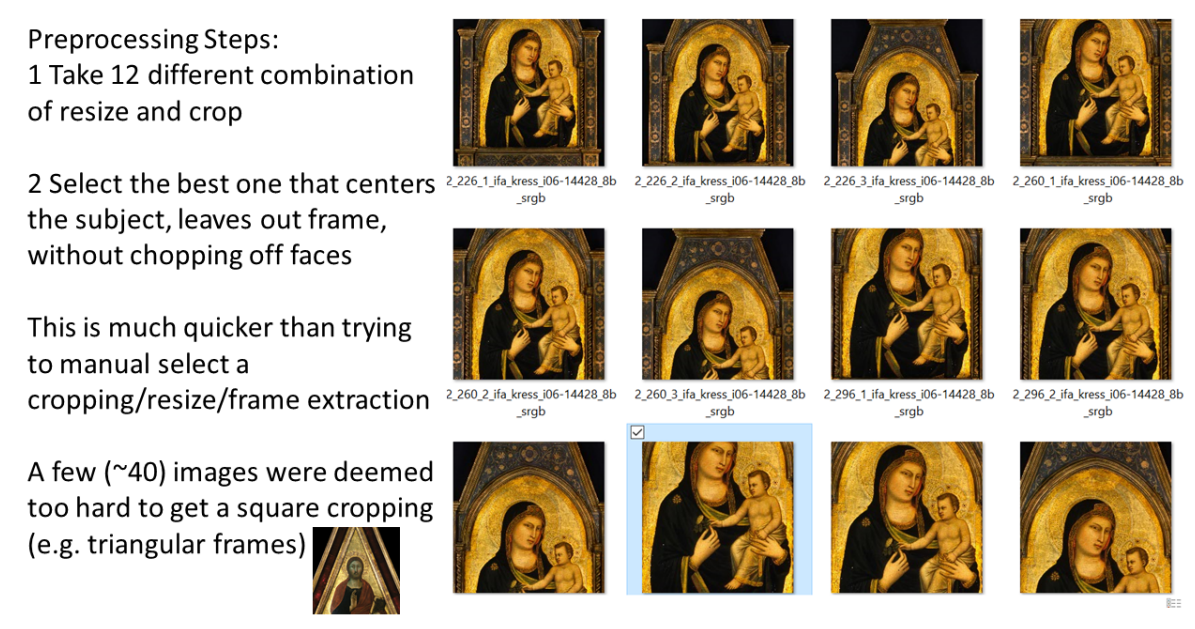

Figure 2: A semi-manual way to crop out frames and/or unwanted background of the digitization. The first three images in the first row are resized to $224 \times 224$ with different offsets from center of the image.

\section{VGG19 - pretrained CNN for object} recognition Extract max-pool convolution layer maps as features

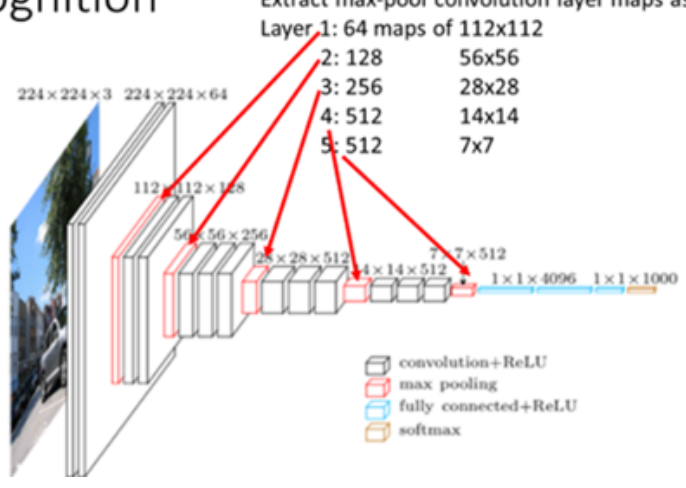

Figure 3: The VGG19 deep network [3] pretrained in 1000 class object recognition.

network, as a general feature detector, extract those features, and feed them into K-means clustering.

The procedure for extracting and processing features was as follows: the 1820 images were run through VGG19 network and the five max-pool activation layers were extracted (Figure 1). However, this produced large number of values $(>140000)$ for each of 1820 rows in a data matrix. Instead of combining all maps and doing dimension reduction on 140,000 plus columns we summarized each feature map separately and then combined the summaries as follows. Each map in each max-pool layer was summarized by taking the norm (i.e., sum square) and scaled by the log function - this left 1 number for each map and a $1820 \times 1472$ data matrix. In other words, the last max pool layer was 512 maps of $7 \times 7$ reduced to 512 values. This avoids mixing together all maps before a decomposition so that each feature map (and each underlying filter) is equally represented.
Moreover, it reflects the notion that $\mathrm{CNN}$ features "tend to encode the spatial presence of some pattern or concept over the different tiles of the feature map" [4] (our emphasis).

\subsection{Predetermined Features}

Convolution network filters are described as feature discovery because the filters are learned and that is what determines which pixel combinations activate nodes. Therefore, for a comparison, we also used a collection of predetermined features that are standard in computer vision and often more appropriate for small tasks or small datasets than a CNN. These include: 1) Histogram of Gradients (HOG) [5] - over six spatial scales (cell sizes $=2,4,8,16,32,64$ ), we took the number of pixels with at least $1 / 3$ of its gradients' magnitude in any direction; 2) entropy - which measures overall randomness of an image; 3 ) two edge detectors (Sobel and Canny) - for which we took the total number of pixels that comprise all edges; and 4) compression ratio - namely how much an image and be compressed as when saving it in JPG format. This produced a $1820 \times 10$ data matrix of predetermined features. Notice that these summaries lose spatial information, which is analogous to the CNN summaries used above. Whereas the $\mathrm{CNN}$ summaries reflect the presence of some undefined feature, here they reflect the presence of some given feature.

\section{RESULTS}

\subsection{Clusters}

After processing the feature maps from the VGG19 network, and transforming those maps into a feature space for cluster, we ran $\mathrm{K}$-means and hierarchical clustering in an exploratory analysis. We first tried to assess the number of clusters using standard K-means using the Silhouette test. The Silhouette test typically showed 2-4 clusters. We also applied a hierarchical clustering (Figure 4) so that we could view different clusters as they might change depending on the depth of the dendrogram starting from the root. For a depth 


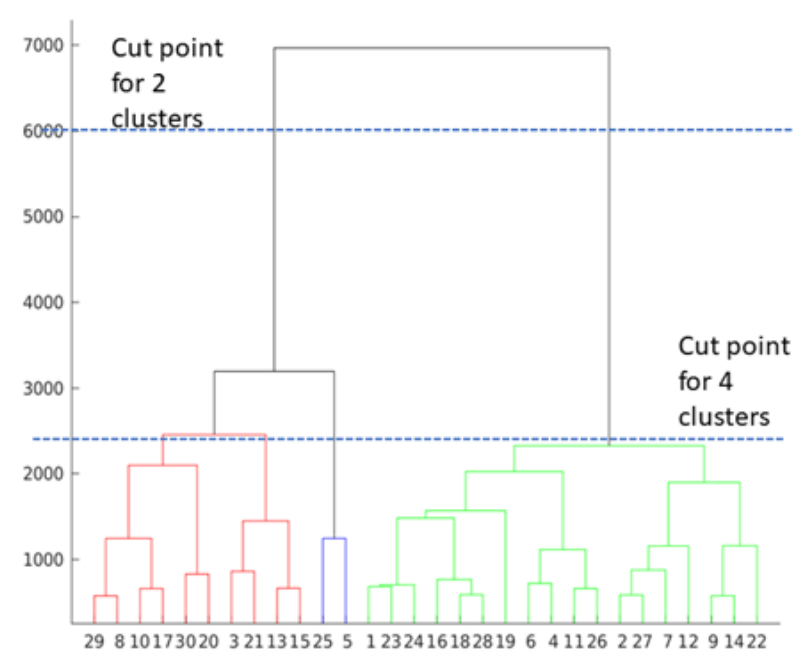

Figure 4: Dendrogram of highest levels of hierarchical clusters.

of 2 clusters we ran t-tests for each individual feature to ask which features have discriminatory information that separate the data into 2 clusters. It turns out that 1297 of the 1472 features have significantly different mean values between the first 2 clusters, with a Bonferroni corrected p-value. As we go from 2 to 4 clusters (ie going down the dendrogram), we found that 819 features had significant corrected p-values. And as we kept going down the tree large numbers of features help discriminate between clusters. Intuitively, we can see subject matter and visual differences in the clusters (see Figure 5), which suggests that the perceptible differences we identify are high level features that are made up of a large number of lower level features in the convolution networks. In other words, there is not going to be a high-level 'women holding baby' feature. Instead, it seems that images with that subject consist of many lower level color, texture, and edge features having to do with patterns of face elements, hair elements, etc. that comprise a women holding a baby.

As a further investigation we utilized the functionality available in the Keras/Tensorflow utilities to look at specific features (e.g. Chollet) [2] We isolated the one feature (layer 3, map 236) that most discriminated the top two clusters in Figures 4 and 5 and the one feature (layer 3, map 245) that most discriminated the last two clusters in Figures 4 and 5. Starting with a random image, gradient ascent was applied to the image to generate an image pattern that would maximize the activation of that feature map. The resulting pattern gives an indication of what kind of pixel combinations a natural image might have related to that pattern. Figure 6 shows the resulting patterns. They show different scales and orientation of repetition and different amounts of saturation or hue, but otherwise it is not obvious how one might identify it. However, one might surmise that 100 s of such features together would discriminate groups of paintings. In future work we will try to take subspaces of features to see if we can characterize clusters at a lower level of description than something like 'woman holding baby'.

\subsection{Other Feature Comparisons}

We applied several Computer Vision techniques to summarize an image. We found that HOG edge summaries are most correlated, on average, with layers $2,3(\sim 0.32-0.42)$ and least with layer 5 ( $\sim 0.08-0.14$ ). For example, the summary of the HOG 16-pixel cell size measurements is most correlated, on average, with middle CNN layer 2 (0.42). On the other hand, edge detection summaries, entropy, and compression ratios are most correlated with lower layers ( 0.43-0.57 among layers 1,2,3).

Within CNN layers at the same level, the absolute correlations averaged from $\sim 0.59$ (layer 2) to 0.11 (layer 5). Between layers, the absolute correlations decrease with difference in layer levels; for example, for layer 1 and layer 2 the average is $\sim 0.53$, but for layer 1 and layer 5 the average is $\sim 0.14$.

We also checked if the summary of feature maps was too coarse by comparing it to principal components for all columns of all feature maps combined one layer at a time. The total feature maps for individual layers form $\mathrm{Nx}(\mathrm{PxHxW})$ matrices, where $\mathrm{HxW}$ is height and width of each feature map in one layer, and $\mathrm{P}$, the number of maps in a layer, is in $\{64,128,256,512,512\}$, and $N=1820$. The summary of features we use for individual layers form $\mathrm{NxP}$ submatrices. We found that the 1st principle component of the summary matrix is highly correlated with (0.6-0.9), but with lower variance than, the 1st component of the full matrix. Thus, the summary matrix does maintain a lot of information in a feature map.

We also examined the CNN output. Although many of the labels are reasonable, many predictions were weak $(<0.5)$ and there was much overlap between images from different clusters. The highest average predictions for the 4 clusters in Figure 5 (top to bottom) are: 1: "altar, throne, book jacket" 2:"book jacket, cloak, vestment, fur coat" 3: "cloak, book jacket" 4: "altar, vestment, book jacket, throne, cloak". Of course, the CNN is only trained on 1000 object categories so one should not expect conceptual matches regarding scenes but more perceptual matches regarding object-like features.

\section{CONCLUSION}

With the success of deep learning for object recognition, we wondered what application it might have to the authorship attribution of historical paintings. Researchers have shown that deep networks can learn to classify original line drawings from copies by defining simple but reasonable features like line thickness [6], or that deep network classification layers can reasonably cluster images with strong color differences [9]. More similar to our work is research that attempts to characterize features of a CNN. One work [10] also used a pretrained $\mathrm{CNN}$, input new object images, extracted features maps, and then used clustering to produce labels based on the objects in a cluster. However, that work used objects in the same classes as the original training set, which ensured that features maps would correspond to obvious image distinctions. In this work, we considered the harder exploratory case of unsupervised learning with no prior conception about what could be a good clustering, and a particular interest in what the convolution layers could help sort out. We hypothesized that the nature of information in convolution layers could inform or challenge humanistic perspectives. Towards that end, we investigated deep network convolution activations for a set of historical paintings with varied subject matters and styles, 


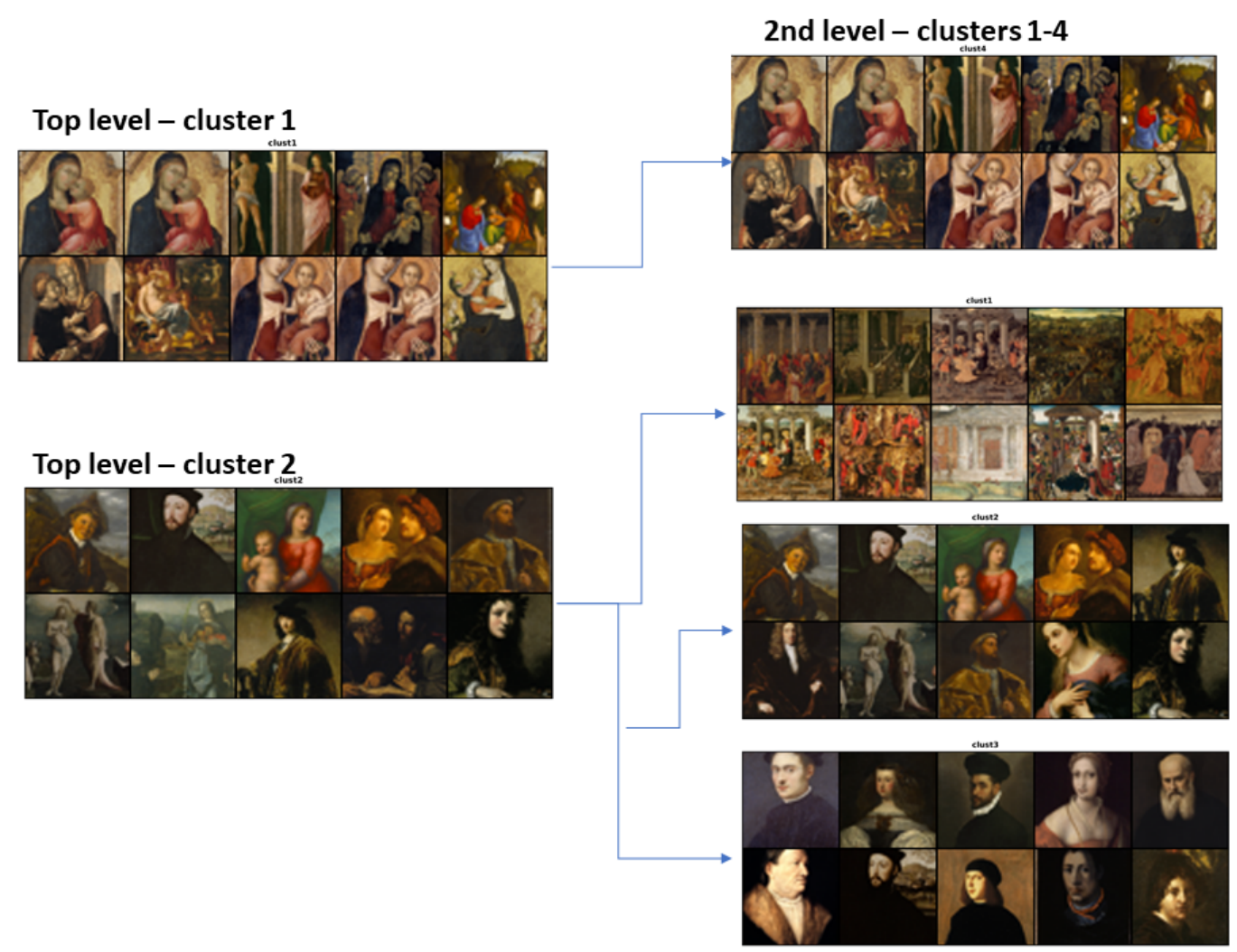

Figure 5: Top 2 clusters and the splits into top 4 clusters from dendrogram. The montages show images in center of cluster. Notice some image were duplicates.

A. Layer $3,236^{\text {th }}$ feature

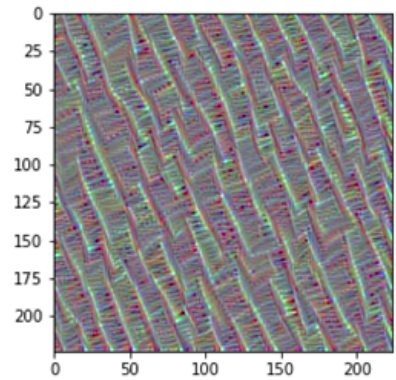

B. Layer $3,245^{\text {th }}$ feature

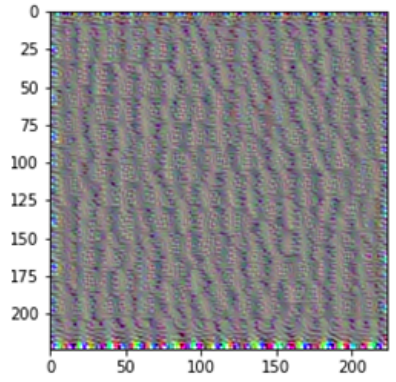

Figure 6: Patterns that were generated to maximize activity for feature maps do not have obvious interpretations. (A) Is for the feature that most separated top two clusters in dendrogram tree (Figures 4, left side Figure 5) and (B) is for the feature that most separated the 3rd and 4th clusters (Figure 4, cut off for 4 clusters, left most; right column of Figure 5).

and then asked what kind of features in the network distinguish these paintings.

We found that features in CNNs can be highly visually discriminating between groups of paintings. However, we do not necessarily

find that paintings are falling into well-defined categories that we would identify as "by style" or "by artist." The CNN features appear to be highly overlapping or related, and pick up on relatively small scale or particular image characteristics, such as lower level color, texture, or edge features. And these features, especially the combination of such features, are hard to articulate as something an art historian would recognize as 'stylistic'. On the other hand, this kind of work is one way of looking at what a computer might consider the elements of visual similarity and difference between pictures, which may or may not align with what art historians consider to be "style."

Finally, it is worth pointing out that convolution network features maps are somewhat analogous to the human visual system in both the nature of the features and object discrimination properties [7]. No one claims it is a biological model, but it helps think about biological functions and how visual perception could be heavily influenced by 'top-down' attentional and semantic knowledge [8]. In a similar manner, for the digital humanist, deep convolution networks can be useful to think about the space of visual features, and the varied processes of using that space. It may or may not directly contribute to our understanding of attribution or style, but it can serve as a foil or as a case study. In this sense, one line 
of future work could further explore how features in a convolution network could be manipulated, post-processed, or mixed, to distinguish paintings in different ways.

\section{ACKNOWLEDGMENTS}

This work used the Extreme Science and Engineering Discovery Environment (XSEDE), supported by National Science Foundation grant number ACI-1548562, with Extended Collaboration and Novel Project support, allocation uop137, on Comet system at San Diego Supercomputer Center.

\section{REFERENCES}

[1] Saltz, Jerry. "Jerry Saltz: Christie's Says This Painting Is by Leonardo. I Really Doubt It." (2017) Vulture, 14 Nov. 2017, www.vulture.com/2017/11/christies-saysthis-painting-is-by-leonardo-i-doubt-it.html

[2] Langmead, Alison, Nygren, Christopher (in preparation). "Leonardo, Morelli, and the Computational Mirror".
[3] Simonyan, Karen, Zisserman, Andrew. (2015). "Very Deep Convolutional Networks for Large-Scale Image Recognition." International Conference on Learning Representations, San Diego, CA, USA, May 7-9, 2015 https://arxiv.org/abs/1409. 1556).

[4] Francois Chollet. (2018) Deep Learning with Python. Manning publications.

[5] Dalal, N. , B. Triggs. (2005) "Histograms of Oriented Gradients for Human Detection", IEEE Computer Society Conference on Computer Vision and Pattern Recognition, Vol. 1 June 2005, pp. 886-893.

[6] Ahmed Elgammal, Yan Kang, Milko Den Leeuw (2018). "Picasso, Matisse, or a Fake? Automated Analysis of Drawings at the Stroke Level for Attribution and Authentication." 32 AAAI Conference on Artificial Intelligence .

[7] Kriegeskorte,Nikolaus (2015). "Deep Neural Networks: A New Framework for Modeling Biological Vision and Brain Information Processing”. Annual Review of Vision Science. 1:1, 417-446.

[8] Gilbert, Charles, Li, Wu. (2013). Top-down influences on visual processing. Nature reviews. Neuroscience. 14. 350-363. 10.1038/nrn3476.

[9] Zaslavsky, Ilya. SUAVE, suave.sdsc.edu/suave-for-art-history/.

[10] Wang, Zhishuai, Zhang, Cihang, Premachandran, Vittal, Yuille, Alan (2016) "Unsupervised learning of object semantic parts from internal states of CNNs by population encoding" https://arxiv.org/pdf/1511.06855.pdf 